\title{
MICROLITIASIS TESTICULAR: ¿EXISTE RELACIÓN CON PATOLOGÍA MALIGNA?
}

\author{
R. RODRÍGUEZ VILLALBA, M. SCARFINI, E. RUIZ CASTAÑÉ, \\ J.M. POMEROL MONSENY
}

Departamento de Andrología. Fundación Puigvert. Barcelona.

Actas Urol Esp. 28 (1): 38-39, 2004

\section{RESUMEN}

MICROLITIASIS TESTICULAR: ¿EXISTE RELACIÓN CON PATOLOGÍA MALIGNA?

El propósito de esta revisión es evaluar la relación de microlitiasis testicular con tumor maligno, para lo que se realizó un estudio retrospectivo de 98 pacientes con diagnóstico histológico de tumor maligno testicular.

El diagnóstico de estos pacientes se realizó ecográficamente, presentando calcificaciones testiculares el $6,02 \%$ (6 pacientes), lo que nos hace concluir que el hallazgo de litiasis testicular no es característico de esta patología.

A propósito de esta revisión, se consultó bibliografía relacionada con el tema, evidenciándose que la conducta ante calcificaciones aisladas en ausencia de tumor sigue siendo controversial.

PALABRAS CLAVE: Microcalcificaciones. Litiasis testicular. Tumor testicular. Ecografia testicular.

\section{ABSTRACT}

TESTICULAR MICROLITHIASIS: DOES EXIST ANY RELATIONSHIP WITH MALIGNANT TUMOUR?

The aim of this revision is to assess the relationship between testicular microlithiasis and malignant tumours. We reviewed 98 charts of patients with pathological diagnosis of testicular malignant tumour.

Diagnosis of testicular tumour was made by ultrasound, and $6.02 \%$ (6 pts.) presented testicular lithiasis, and this finding makes us conclude that this is not a characteristic feature of this entity.

We also reviewed bibliography related to this theme, and conclude that the management of microlithiasis remains being controversial.

KEY WORDS: Testicular microlithiasis. Testicular neoplasms. Testicular ultrasound.

$\mathrm{L}$ as microcalcificaciones testiculares han sido relacionadas con diferentes patologías inflamatorias y tumorales, sin que exista actualmente un consenso acerca de su potencial maligno y de la conducta clínica a seguir ante ellas.

\section{OBJ ETIVO}

Determinar la presencia o no de litiasis en la ecografía testicular preoperatoria de pacientes con diagnóstico histopatológico de tumor maligno.

\section{MATERIALES Y MÉTODOS}

Se realizó un estudio retrospectivo de 100 pacientes escogidos al azar con diagnóstico de tumor testicular que acudieron a nuestro centro desde el año 1995 al 2000, con un seguimiento medio de 24 meses. Se excluyeron del grupo 2 pacientes con datos insuficientes en la historia clínica para ser evaluados.

\section{RESULTADOS}

La media de edad del grupo $(n=98)$ fue de 31,51 años (rango: 14-68). Los tumores de testículo derecho fueron más frecuentes (57 pacientes que equivalen al 60,64\%) que el izquierdo, en el que se presentaron el $39,36 \%$ de los tumores (37 pacientes), siendo 4 del total bilaterales ( 3 seminomas típicos y 1 tumor mixto). 
Los diagnósticos patológicos fueron:

44 casos $(44,89 \%)$ de seminoma típico.

39 casos $(39,79 \%)$ de tumor mixto.

4 casos $(4,09 \%)$ de carcinoma embrionario.

4 casos $(4,09 \%)$ de teratoma.

5 casos $(5,10 \%)$ de tumor germinal intratubular.

1 caso $(1,02 \%)$ de carcinoma in situ.

1 caso $(1,02 \%)$ de tumor de células de Leydig.

32 pacientes $(32,65 \%)$ acudieron a consulta por dolor testicular como síntoma predominante.

Sólo 6 pacientes $(6,12 \%)$ de toda la serie presentaron microlitiasis testicular en la ecografía testicular. El diagnóstico anatomopatológico en ellos fue: tumor mixto en 2 de los casos, tumor de células germinales en otros 2 casos, seminoma en 1 caso y teratoma en 1 caso.

\section{DISCUSIÓN}

La microlitiasis testicular es un hallazgo ultrasonográfico relativamente poco frecuente en tumores testiculares, no existiendo relación con el tipo histológico del tumor.

Las calcificaciones intratesticulares son producidas por la formación de microlitos de células degenerativas en los túbulos seminíferos y han sido relacionadas con diferentes tipos de entidades: flebolitos, calcificaciones vasculares y granulomas espermáticos ${ }^{1}$.

Además de revisar esta serie de pacientes con diagnóstico de tumor testicular para evaluar la presencia o no de calcificaciones en la ecografía diagnóstica, hemos hecho una revisión de la literatura donde se describen diferentes series que relacionan características ecográficas con patología de base (orquitis, epididimitis, tumores) ${ }^{1,2}$.

Existen controversias en el punto de la necesidad de seguimiento ecográfico ante un diagnóstico de microcalcificaciones testiculares ${ }^{2}$.

Otite U et al. ${ }^{2}$, después de evaluar un grupo de 3.026 pacientes referidos por dolor, aumento de volumen testicular o infertilidad, concluyen un riesgo relativo de tumor testicular en presencia de microcalcificaciones de 13,2.

En cambio, Peterson AC et al. ${ }^{3}$ concluyen, como en nuestro caso, que las microcalcificaciones testiculares son un hallazgo común en hombres asintomáticos que pueden no estar relacionadas con tumores malignos de testículo.

Otros autores como von Eckardstein ${ }^{4}$, luego de estudiar 1.701 ecografías con patologías de base (infertilidad, tumor contralateral, testes atróficos) y compararlos con 198 voluntarios normales, concluyeron que el diagnóstico de microlitiasis, especialmente si está presente en testes atróficos, requiere de una biopsia diagnóstica o, al menos, ecografías testiculares seriadas para descartar patología.

Bach $\mathrm{AM}^{5}$, reportó una serie de 528 pacientes, de los cuales 48 (9\%) presentaban microlitiasis en la ecografía testicular, siendo 13 (27\%) de ellos diagnosticados de tumor testicular.

En nuestra serie existe un 6,12\% de incidencia de microcalcificaciones asociadas a tumor testicular, por lo que no lo consideramos como un hallazgo característico de tumor testicular, y, por lo tanto, no recomendamos ningún tipo de seguimiento ecográfico o procedimiento invasivo diferente al examen físico anual de control.

\section{REFERENCIAS}

1. BUSHBY LH, MILLER FN, ROSAIRO S, CLARKE JL, SIDHU PS.: Scrotal calcification: ultrasound appearances, distribution and aetiology. Br J Radiol 2002 mar; 75 (891): 283-288.

2. OTITE U, WEBB JA, OLIVER RT, BADENOCH DF, NARGUND VH.: Testicular microlithiasis: is it a benign condition with malignant potential?. Eur Urol 2001 nov; 40 (5): 538-542.

3. PETERSON AC, BAUMAN JM, LIGHT DE, MCMANN LP, COSTABILE RA.: The prevalence of testicular microlithiasis in an asymptomatic population of men 18 to 35 years old. J Urol 2001 dec; 166 (6): 20612064.

4. VON ECKARDSTEIN S, TSAKMAKIDIS G, KAMISCHKE A, ROLF C, NIESCHLAG E.: Sonographic testicular microlithiasis as an indicator of premalignant conditions in normal and infertile men. $J$ Androl 2001 sep-oct; 22 (5): 818-824.

5. BACH AM, HANN LE, HADAR O, SHI W, YOO HH, GIESS CS, SHEINFELD J, THALER H.: Testicular microlithiasis: what is its association with testicular cancer?. Radiology 2001 jul; 220 (1): 70-75.

6. DEROGEE M, BEVERS RF, PRINS HJ, JONGES TG, ELBERS FH, BOON TA.: Testicular microlithiasis, a premalignant condition: prevalence, histopathologic findings, and relation to testicular tumor. Urology 2001 jun; 57 (6): 1133-1137.

Dra. Rosana Rodríguez Villalba

Departamento de Andrología

Fundación Puigvert

C/ Cartagena, 340

08025 Barcelona

(Trabajo recibido el 4 marzo 2003) 